\title{
Morphology of Holographic Polymer Dispersed Liquid Crystal Reflection Gratings Written in Thiol-ene and Acrylate Polymer Hosts: Part I-Grating Formation
}

\author{
D.W. Tomlin, ${ }^{* *}$ L.V. Natarajan,* V.P. Tondiglia,* R.L. Sutherland,* and T.J. Bunning
}

Air Force Research Laboratory, Materials and Manufacturing Directorate, WPAFB, OH 45433

*Science Applications International Corporation, 4031 Col. Glenn Highway, Dayton, OH 45431

**UES, Inc, 4401 Dayton-Xenia Road, Dayton, OH 45432

Holographic polymer-dispersed liquid crystals (H-PDLC) are formed by the non-homogeneous spatial illumination of monomer/LC mixtures. The anisotropic distribution of polymer and LC-rich lamellae give rise to a periodic refractive index modulation, which can be electrically modulated. The focus of research in H-PDLCs has been on highly cross-linked acrylate polymer systems in which a large number of additives are typically required. These additives are potential sources of contamination in the LC droplets causing a decrease in clearing temperature and order parameter. Multifunctional acrylates as hosts for H-PDLC gratings have some drawbacks due to low final monomer conversion caused by early gelation. This can cause shrinkage, blue shifting of the notch, asymmetric notch shapes, and refractive index chirping. The large distribution of droplet shapes and interconnectivity typical in a fast gelling system also diminishes the electrical switching, as well as increases in unwanted scatter due to increased interfacial area.

Thiol-ene polymers are well known for their role in the preparation of UV curable coatings and adhesives and are more elastic. These polymers are formed by the combination of step growth and free radical reactions between multifunctional aliphatic thiols and vinyl monomers. Due to the late gelation, polymerization can be driven to almost 100\% conversion, thus reducing the non-uniform shrinkage relative to multifunctional acrylate monomers. Both thiol-ene and acrylate monomers are used as hosts for the phase separation of nematic liquid crystals in conventional polymer-dispersed liquid crystals. In both cases, high molecular weight polymer is formed and therefore phase separation takes place resulting in a two-phase composite whose optical properties can be electrically modulated. Although a wide range of nematic droplet morphologies can be produced depending on intensity and LC concentration, there are typically large differences in the two-phase morphologies between monomer types. The acrylate systems typically possess a heterogeneous droplet size and shape (non-spherical) distribution (Figure 1(a), 1(b)) whereas thiol-ene systems exhibit spherical and monodisperse domains (Figure 2(a), 1(b)). These differences are due to differences in the molecular weight (MW) evolution as a function of time between two systems. In acrylates, where high MW polymer is formed immediately, gelation typically precedes phase separation. In thiol-enes, MW increases in a step-wise fashion, and classic liquid-liquid phase separation occurs.

To investigate the interior morphology of these H-PDLC gratings, samples were investigated in detail employing bright field transmission electron microscopy (BF TEM). The BF TEM images were obtained on a FEI CM200 FEG TEM on unstained and stained 50-60 nm sections. Ultramicrotomy was performed at room temperature on a Reichert Ultracut utilizing a variety of DiATOME diamond knives, which will be discussed in more detail in Part II. The BF TEM images in Figure 1 and Figure 2 reveal a different morphology for the thiol-ene samples. The thiol-ene 
system's interface was very smooth, unlike the acrylate system, where very irregular surfaces are observed between the droplets and polymer.

We have demonstrated the H-PDLC reflection gratings can be recorded in thiol-ene based polymer systems. The combination of free radical and step growth polymerization offers several advantages for the optical and electro-optical performance of H-PDLC gratings over conventional free radical polymerization of highly functional acrylates. These multifunctional acrylates polymerize via a chin growth mechanism where monomer is successively added rapidly to a growing chain. High MW polymer is formed almost immediately and the monomer concentration decreases steadily with time. In a high functionality chain growth system, diffusion limited reaction kinetics dominate early and irregular shaped domains are formed due to local heterogeneity of the phase separation process. The LC rich phase resides in these interstitial areas. In the case of the thiol-ene system, though the initial steps involve free radical addition processes, a step growth mechanism is dominant. The MW of the polymer increases slowly, as the number of monomers increases above a critical concentration, a liquid-liquid phase separation occurs. Because both phases are liquid and the viscosity is low, the discontinuous phase typically is spherical due to surface tension effects. As the reaction progresses, at some point the polymer/monomer phase vitrifies and traps the spherical domains.

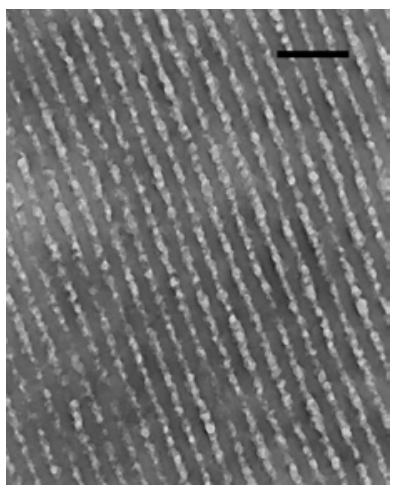

Figure 1: (a)

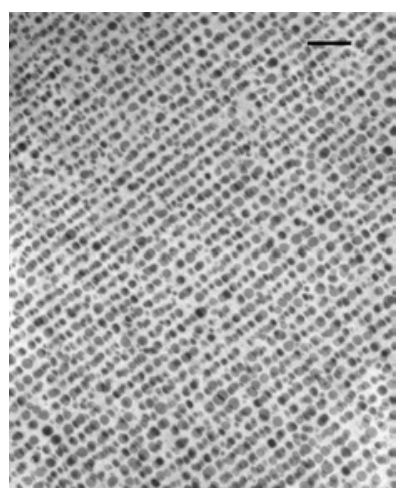

Figure 2: (a)

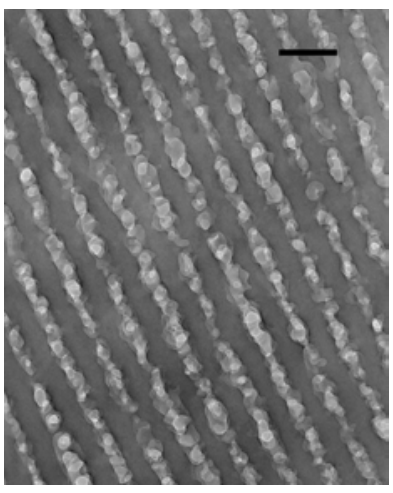

(b)

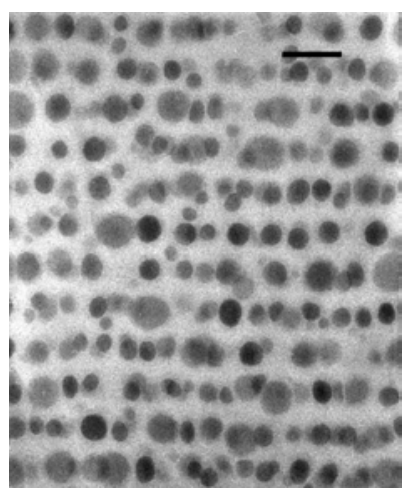

(b)

FIG. 1: Actual spacing is $154 \mathrm{~nm}$ (a) acrylate grating scale bar is $500 \mathrm{~nm}$; (b) acrylate grating scale bar is $200 \mathrm{~nm}$.

FIG. 2: Actual spacing is $150 \mathrm{~nm}$ (a) thiol-ene grating scale bar is $500 \mathrm{~nm}$; (b) thiol-ene grating scale is $200 \mathrm{~nm}$ 